\title{
High-intensity exercise improves pulmonary function and exercise tolerance in a patient with TSC-LAM
}

\begin{abstract}
Introduction: While exercise has been shown to improve respiratory symptoms, exercise tolerance, and bone mineral density in many populations, no supervised exercise training interventions have been undertaken in patients with lymphangioleiomyomatosis (LAM).

Material and methods: One patient with TSC-LAM (tuberous sclerosis complex lymphangioleiomyomatosis) participated in two weekly sessions ( $50-60 \mathrm{~min}$ ) of supervised aerobic exercise at $80-85 \%$ heart rate max for one year. Treadmill ergometry $\left(\mathrm{VO}_{2 \text { peak }}\right)$, spirometry ( $\mathrm{FEV}_{1}, \mathrm{FVC}_{1}, \mathrm{FEV}_{1} / \mathrm{FVC}$, peak flow), and bone mineral density testing were performed prior to every 3 months.

Results: After one year of supervised aerobic exercise training we saw dramatic increases in the patient's $\mathrm{VO}_{2 \max }(20 \%), \mathrm{FEV}_{1}$ (9.5\%), $\mathrm{FEV}_{1} / \mathrm{FVC}(9.1 \%)$ and peak flow $(47 \%)$.

Conclusions: The results from this study indicate that supervised exercise training can improve exercise tolerance and pulmonary function in a patient with lymphangioleiomyomatosis. Further research is needed, including longitudinal studies with larger sample sizes, to determine long-term effects and consistency of these findings. Aerobic exercise may offer a viable alternative or compliment to pharmacological interventions in the treatment of lymphangioleiomyomatosis. We show that high-intensity exercise training can markedly and safely improve pulmonary function in a patient with TSC-LAM. While we did not record quality of life or mood states, our patient did report improved self-confidence as well as enhanced mood.
\end{abstract}

Key words: tuberous sclerosis, exercise, lymphangioleiomyomatosis

Adv Respir Med. 2020; 88: 356-359

\section{Introduction}

Approval was obtained from the Committee for Protection of Human Subjects at the University of Houston and the Institutional Review Board at the University of Texas Health Science Center (UTHSC) and at the University of Central Arkansas. Written informed consent was obtained from our patient prior to data collection. A 29-year-old female with tuberous sclerosis complex lymphangioleiomyomatosis (TSC-LAM; TSC diagnosed in 1988, age 5; LAM in 2004, age 21) with a history of related complications (more than twenty pneumothoraces, resulting in bilateral pleurodesis and pleurectomy; angiomyolipomas resulting in partial nephrecotmy) was referred from the UTHSC LAM Clinic by her LAM specialist. Her last pneumothorax was approximately five years prior to her inclusion in this trial (age 23), when she received her bilateral pleurectomy/pleurodesis. The psychological state of the patient was motivated but she was lacking in confidence in her ability to perform even moderate exercise (e.g., more than walking at a slow pace on a treadmill for more than a few minutes). The patient was taking sirolimus for 3 years, switching to afinitor 4 years ago due to attenuation (sirolimus benefits were plateauing) of sirolimus on disease. The patient did not have any brain lesions, nor had she ever experienced any episodes of epilepsy.

\section{Material and methods}

Pulmonary function tests (PFTs), a graded exercise test (GXT), and bone mineral density (BMD) were assessed prior to beginning exercise training 


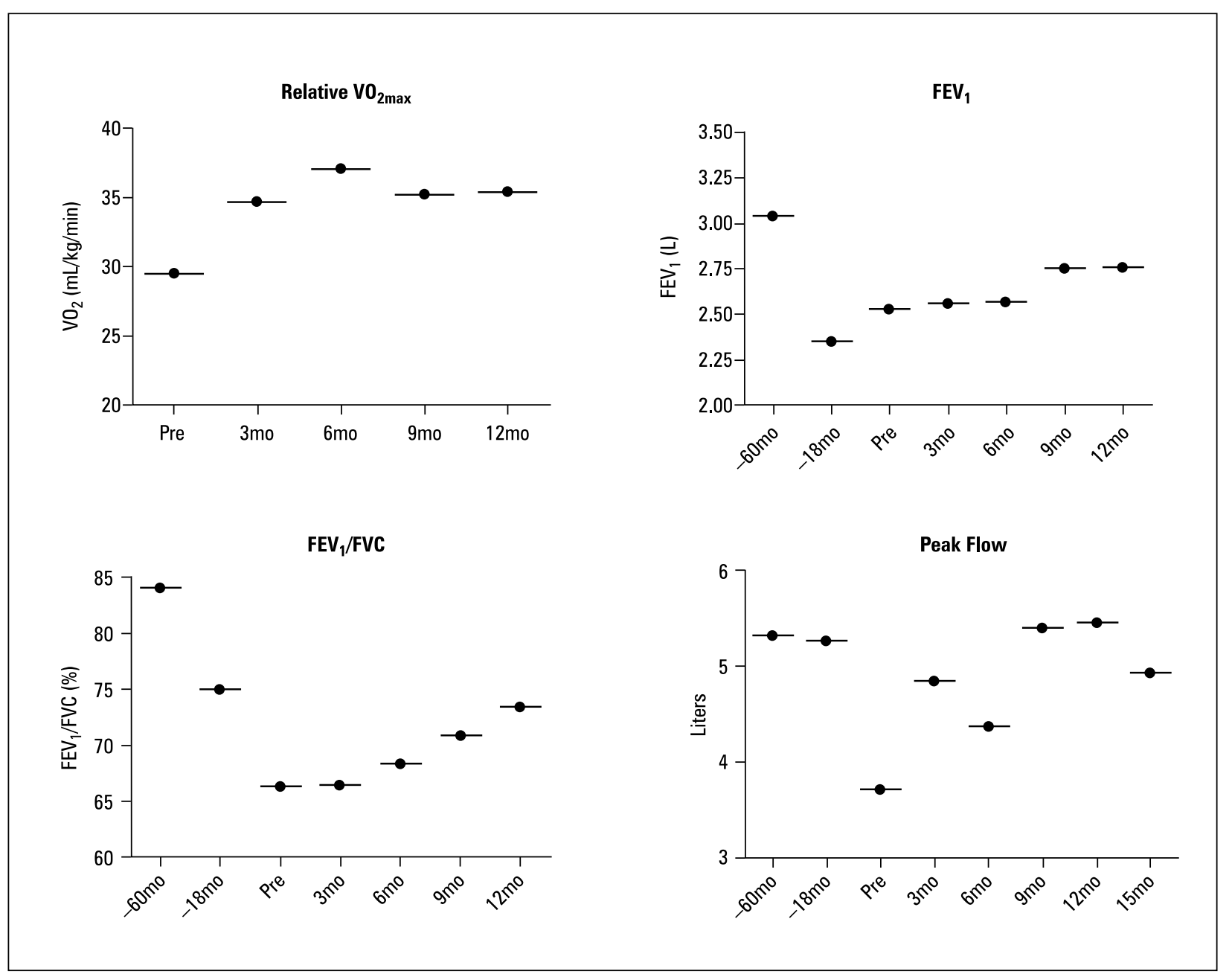

Figure 1. Oxygen uptake $\left(\mathrm{VO}_{2 \max }\right)$ and pulmonary function tests

(pre) and every three months. Twice weekly the patient reported for supervised exercise training (60 min/session), consisting of high-intensity aerobic (primarily treadmill/track running/sprinting) and resistance training. Patient performed a GXT to volitional fatigue to determine $\mathrm{VO}_{2 \max }$ using a Woodway Desmo treadmill (Waukesha, WI, USA) and a Quark CPET metabolic cart (Cosmed, Rome, Italy). PFTs were assessed using the Quark CPET metabolic cart. A Discovery QDR-4500 for Windows (Hologic, Inc., Bedford, MA, USA) dual X-ray absorptiometry (DXA) scanner was used to assess BMD. Tests were completed in room air without bronchodilator. PFTs prior to baseline (-60 months, -18 months) were taken from patient records.

Our patient demonstrated marked improvements in $\mathrm{VO}_{2 \max }$ (Figure 1A), $\mathrm{FEV}_{1}$ (forced expiratory volume in 1 second) and $\mathrm{FEV}_{1} / \mathrm{FVC}$ (forced vital capacity) \% (Figure 1B and 1C), and peak flow (Figure 1D). Whole body and spine BMD also slightly improved or remained high despite already-high levels (Table 1). Forced vital capacity remained steady from baseline (Table 1). Patient maintained bodyweight (61.4-64 kg) and body fat (21.9-27.2\%) pre to post. Peak flow improved by $47 \%$; relative and absolute $\mathrm{VO}_{2 \max }$ improved by $20 \%$ and $23.4 \%$, respectively. We do not have pulmonary function tests for the patient prior to her initial use of either sirolimus or following cessation of the drug. The baseline (-60 months) were taken approximately one year prior to the patient switching to Afinitor and the -18 months measures were the most recent PFT measures taken prior to her being seen in our laboratory.

\section{Discussion}

Lymphangioleiomyomatosis is a rare, multisystem disease affecting women of child-bearing age almost exclusively and can occur sporadically or as a manifestation of TSC-LAM [1]; indeed, LAM occurs in at least $40 \%$ of women with TSC [2]. Severe cases can lead to respiratory failure. Spontaneous pneumothoraces are common and 
Table 1. Pulmonary function tests, $\mathrm{VO}_{2 \max }$ and bone mineral density

\begin{tabular}{|c|c|c|c|c|c|c|c|}
\hline Measure & -60 months & -18 months & Pre & 3 months & 6 months & 9 months & 12 months \\
\hline $\mathrm{FEV}_{1} / \mathrm{FVC}[\%]$ & 84.0 & 75.0 & 66.3 & 66.4 & 68.4 & 70.9 & 73.4 \\
\hline FVC [L] & 4.33 & 3.71 & 3.82 & 3.88 & 3.80 & 3.97 & 3.82 \\
\hline $\mathrm{FEV}_{1}[\mathrm{~L}]$ & 3.04 & 2.35 & 2.53 & 2.56 & 2.57 & 2.75 & 2.76 \\
\hline Peak flow $[L]$ & 5.32 & 5.26 & 3.71 & 4.85 & 4.36 & 5.4 & 5.45 \\
\hline $\mathrm{VO}_{2 \max }[\mathrm{mL} / \mathrm{kg} / \mathrm{min}]$ & & & 29.43 & 34.66 & 37.02 & 35.23 & 35.34 \\
\hline $\mathrm{VO}_{2}[\mathrm{~L} / \mathrm{min}]$ & & & 1833 & 2160 & 2351 & 2254 & 2262 \\
\hline DXA, whole body $\left(\mathrm{g} / \mathrm{cm}^{2}\right)$ & & & 1.3 & 1.277 & 1.31 & 1.275 & 1.308 \\
\hline DXA, lumbar spine $\left(\mathrm{g} / \mathrm{cm}^{2}\right)$ & & & 1.326 & 1.346 & 1.31 & 1.337 & 1.299 \\
\hline
\end{tabular}

$\mathrm{FEV}_{1}$ — forced expiratory volume in 1 second; FVC — forced vital capacity

are often the primary event leading to diagnosis and in most cases clinical events existed prior to diagnosis with one-third of patients exhibiting normal spirometry and obstruction evident in $57 \%$ of patients [3]. Despite great advances in diagnosis and patient management over the past two decades, treatment options are limited. As such, lifestyle choices and treatment options should be made as early as possible [2]

Sirolimus targets the mammalian target of rapamycin (mTOR) pathway and has been tremendously effective in improving pulmonary function in the majority of patients with LAM [4]. However, once treatment is stopped improvement disappears, and sirolimus appears to have a much greater effect in reducing extrapulmonary masses rather than pulmonary cysts [5]. Further, angiomyolipomas increase once therapy is stopped [6] and LAM is recurrent following lung transplant [7]. $\mathrm{FEV}_{1}$ is considered the most clinically-relevant marker in determining disease severity [8]; patients with $\mathrm{FEV}_{1}<30 \%$ are often placed on transplant list. Improved $\mathrm{FEV}_{1}$ could translate into reduced disease severity, potentially increasing time-to-transplant. A $100 \mathrm{~mL}$ increase in $\mathrm{FEV}_{1}$ has been shown to be clinically important in COPD as this can be perceived by patients [9], and Westwood determined that a $100 \mathrm{~mL}$ increase in $\mathrm{FEV}_{1}$ was associated with improved health status [10]. Our patient exhibited an elevated and sustained $\mathrm{FEV}_{1}$ of $230 \mathrm{~mL}$ (10\% increase, Table 1) beginning at 6 months. Exercise tolerance may be as clinically relevant as $\mathrm{FEV}_{1}[11]$, as individuals able to perform even moderate physical activity would likely have a much-improved quality of life. Here we show an improvement in pulmonary function and exercise tolerance $\left(\mathrm{VO}_{2}\right)$.

Using exercise to treat women with LAM or TSC-LAM is novel. To date, only one study has examined the role of exercise in women with LAM [12]. While that study used exercise, only moderate exercise training was prescribed, and only 12 weeks ( 3 months) of exercise training was undertaken. Here we report on increased pulmonary function in a 29-year-old female with TSC-LAM following one year of intense exercise training. $\mathrm{FEV}_{1}$ and peak flow improved at three and six months, with large increases after nine and twelve months (Figure 1A, D). $\mathrm{VO}_{2}$ improved steadily, peaking at 6 months and remaining elevated. No decline in pulmonary function was seen after one year, unlike sirolimus [4]. These remarkable improvements demonstrate the potential of exercise to improve lung function in women with LAM. Increases were much more dramatic 9-12 months than 3-6 months after beginning the exercise intervention, with levels continuing to rise at one year. Our patient is continuing to exercise to determine if these measures remain elevated past one year. This is the first study in the literature that demonstrates the use of high-intensity exercise to treat LAM. This presents the possibility of extending time-to-transplant with minimal, non-invasive intervention. As $\mathrm{VO}_{2}$ is correlated with disease severity [11], improved $\mathrm{VO}_{2}$ translates clinically and physiologically for patients with LAM.

We are presently preparing a manuscript examining a larger $(n=8)$ group of LAM (seven sporadic LAM and one TSC-LAM) patients examining the positive benefits of exercise training on pulmonary function and bone mineral density at $3,6,9$, and 12 month intervals. While the results of that study demonstrated marked improvements, this patient exhibited even greater results following high-intensity (as opposed to moderateto moderately-high intensity) exercise training. Although quite deconditioned, the patient in this present study was remarkably healthy aside 
from the pulmonary issues associated with TSCLAM and was thus able to eventually participate in high-intensity exercise. Indeed, the patient's pulmonary function $\left(\mathrm{FEV}_{1}\right.$, peak flow) showed the greatest improvements after she had been training for more than six months, indicating that the patient needed to be conditioned before she could be trained using high-intensity exercise training. LAM and TSC-LAM patients undergoing pulmonary rehabilitation should consider longer-term training programs that will allow them to become conditioned to a level such that they can participate in rigorous (high-intensity) exercise programs to improve their gains made during exercise training.

\section{Conflict of interest}

The authors declare no conflicts of interest.

\section{References:}

1. Sato T, Seyama K, Kumasaka T, et al. A patient with TSC1 germline mutation whose clinical phenotype was limited to lymphangioleiomyomatosis. J Intern Med. 2004; 256(2): 166-173, doi: 10.1111/j.1365-2796.2004.01356.x, indexed in Pubmed: 15257730.

2. Cudzilo CJ, Szczesniak RD, Brody AS, et al. Lymphangioleiomyomatosis screening in women with tuberous sclerosis. Chest. 2013; 144(2): 578-585, doi: 10.1378/chest.12-2813, indexed in Pubmed: 23539171.

3. Ryu JH, Moss J, Beck GJ, et al. The NHLBI lymphangioleiomyomatosis registry: characteristics of 230 patients at enroll- ment. Am J Respir Crit Care Med. 2006; 173(1): 105-111, doi: 10.1164/rccm.200409-1298OC, indexed in Pubmed: 16210669.

4. Gupta N, Lee HS, Young LR, et al. Efficacy and safety of sirolimus in lymphangioleiomyomatosis. N Engl J Med. 2011; 364(17): 1595-1606, doi: 10.1056/NEJMoa1100391, indexed in Pubmed: 21410393.

5. Taillé C, Borie R, Crestani B. Current management of lymphangioleiomyomatosis. Curr Opin Pulm Med. 2011; 17(5): 374-378, doi: 10.1097/MCP.0b013e328349ac8c, indexed in Pubmed: 21760507.

6. McCormack FX, Gupta N, Finlay GR, et al. Sirolimus for angiomyolipoma in tuberous sclerosis complex or lymphangioleiomyomatosis. N Engl J Med. 2008; 358(2): 140-151, doi: 10.1056/NEJMoa063564, indexed in Pubmed: 18184959.

7. Bittmann I, Rolf B, Amann G, et al. Recurrence of lymphangioleiomyomatosis after single lung transplantation: new insights into pathogenesis. Hum Pathol. 2003; 34(1): 95-98, doi: 10.1053/hupa.2003.50, indexed in Pubmed: 12605373.

8. Swigris JJ, Lee HS, Cohen M, et al. St. George's Respiratory Questionnaire has longitudinal construct validity in lymphangioleiomyomatosis. Chest. 2013; 143(6): 1671-1678, doi: 10.1378/chest.12-0161, indexed in Pubmed: 23328755.

9. Donohue JF. Minimal clinically important differences in COPD lung function. COPD. 2005; 2(1): 111-124, doi: 10.1081/copd200053377, indexed in Pubmed: 17136971.

10. Westwood M, Bourbeau J, Jones PW, et al. Relationship between FEV1 change and patient-reported outcomes in randomised trials of inhaled bronchodilators for stable COPD: a systematic review. Respir Res. 2011; 12: 40, doi: 10.1186/14659921-12-40, indexed in Pubmed: 21477298.

11. Taveira-DaSilva AM, Stylianou MP, Hedin CJ, et al. Maximal oxygen uptake and severity of disease in lymphangioleiomyomatosis. Am J Respir Crit Care Med. 2003; 168(12): 14271431, doi: 10.1164/rccm.200206-593OC, indexed in Pubmed: 12958050.

12. Araujo MS, Baldi BG, Freitas CSG, et al. Pulmonary rehabilitation in lymphangioleiomyomatosis: a controlled clinical trial. Eur Respir J. 2016; 47(5): 1452-1460, doi: 10.1183/13993003.01683-2015, indexed in Pubmed: 26917604. 